\title{
A large accumulation of metallic foreign bodies in the stomach
}

\author{
DAVID J. KIKOLER, DO \\ DAN L. DUBERSTEIN, DO \\ ALLEN J. ZAGOREN, DO \\ EARL ROZEBOOM, MD
}

\section{A routine $x$-ray examination} showed a fishhook lodged in the esophagus of an asymptomatic 68-year-old man. Further examination revealed multiple foreign objects in his stomach. In all, 82 metallic objects were removed endoscopically. The authors believe that this is the largest accumulation of foreign objects to be removed by this procedure and that endoscopy, properly performed, is a safe and effective alternative to laparotomy. However, surgery remains a safer procedure in some situations.

(Key words: Foreign bodies, stomach, metallic bezoars)

Foreign objects in the gastrointestinal tract may accumulate in the stomach or small intestine, causing complications, such as proteinlosing enteropathy, hemorrhage, steatorrhea, malabsorption, perforation, and obstruction. Most accumulations are classified as either trichobezoars (hairballs) or phytobezoars (foodballs, particularly vegetable matter). There

\footnotetext{
From the Section of Gastroenterology, Department of Internal Medicine (Drs Kikoler and Duberstein) and Department of Surgery (Dr Zagoren), Des Moines General Hospital, Des Moines, Iowa. Dr Rozeboom is in Family Practice, Madison County Memorial Hospital, Winterset, Iowa.

Reprint requests to David J. Kikoler, DO, Central Iowa Gastroenterology, 4001 Ingersoll, Des Moines, IA 50312-2794.
}

are also hard concretions known as bezoar stones, and metallic bezoars. ${ }^{1}$ Most large accumulations are removed surgically. ${ }^{2}$

We present the case of a large accumulation of metallic objects removed endoscopically. Upper gastrointestinal endoscopy for foreign body removal appears to be a safe and effective alternative to laparotomy in appropriate cases.

\section{Report of case}

A 68-year-old man was seen at an outpatient surgical clinic, after he was noted on chest $\mathrm{x}$ ray examination to have a fishhook in his esophagus (Figures 1 and 2). The x-ray examination was made routinely after results of a tuberculin skin test proved to be positive.

Plain abdominal films showed multiple foreign objects clustered in the stomach with some characteristic outlines of commonly recognized foreign bodies (Figure 3). Remarkably, the patient was asymptomatic. He had a history of schizophrenia and lived in a group setting.

Upper gastrointestinal endoscopy revealed a fishhook that was lodged, but not imbedded, in the upper esophagus. It was removed by use of common endoscopic polypectomy snares. Attention was then turned to the foreign objects within the stomach. Over a period of about 4 hours, various rat-tooth forceps and snares were used to endoscopically remove the remain- 


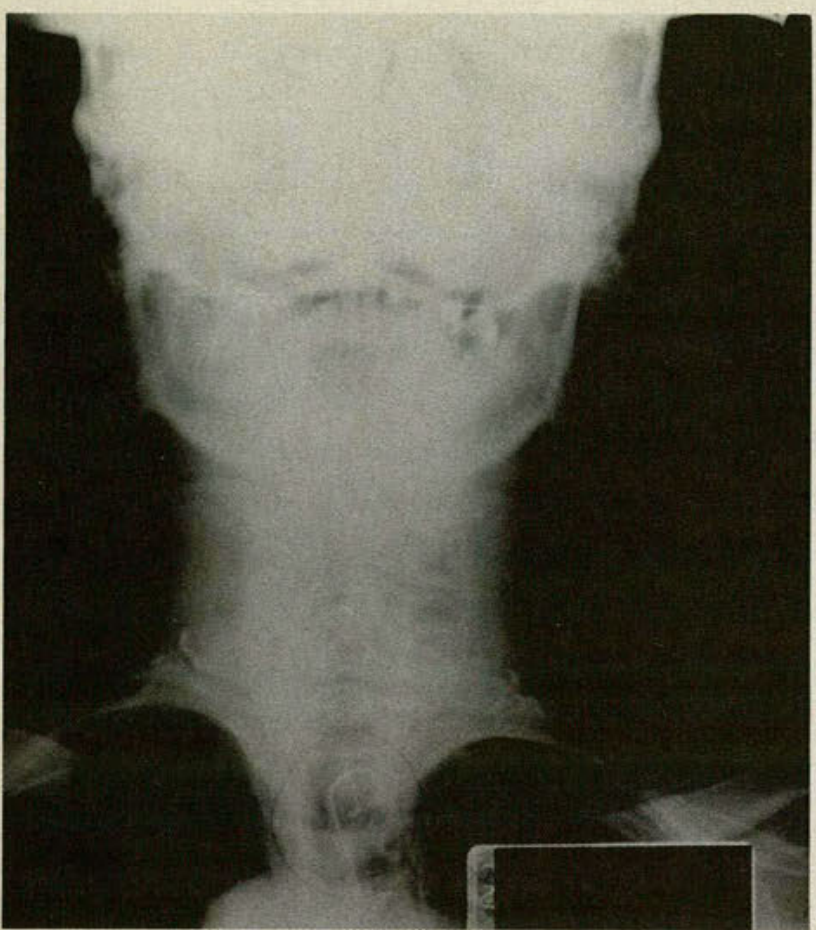

Figure 1. Fishhook in esophagus.

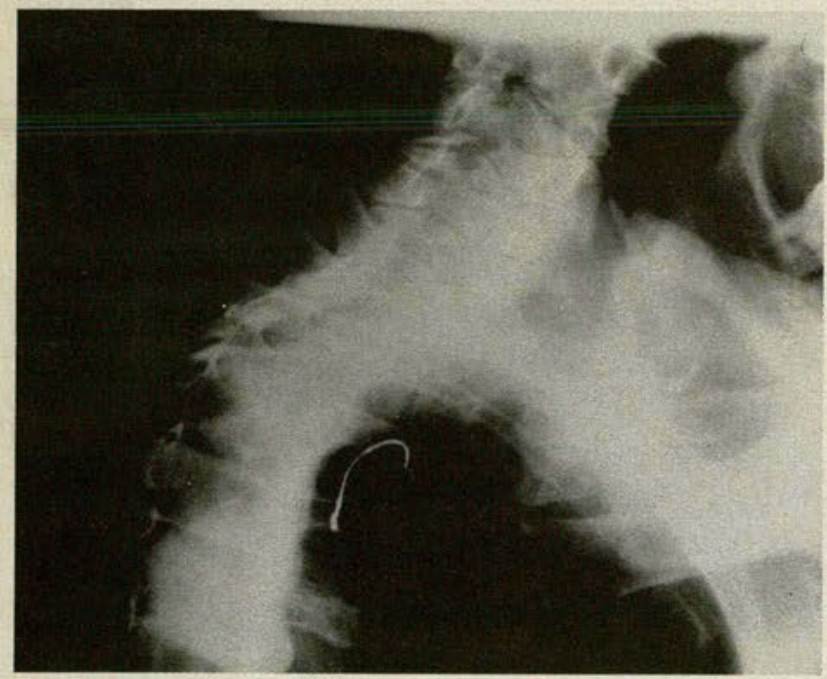

Figure 2. Lateral view of chest showing fishhook in esophagus.

ing 81 foreign objects (Table and Figure 4). An overtube was used to ensure safe removal of the sharper objects. The contents weighed about $405 \mathrm{~g}$ in total. To our knowledge, this is the largest accumulation of foreign objects to be removed endoscopically.

The patient was given medazolam hydrochloride intravenously periodically to allay anxi-

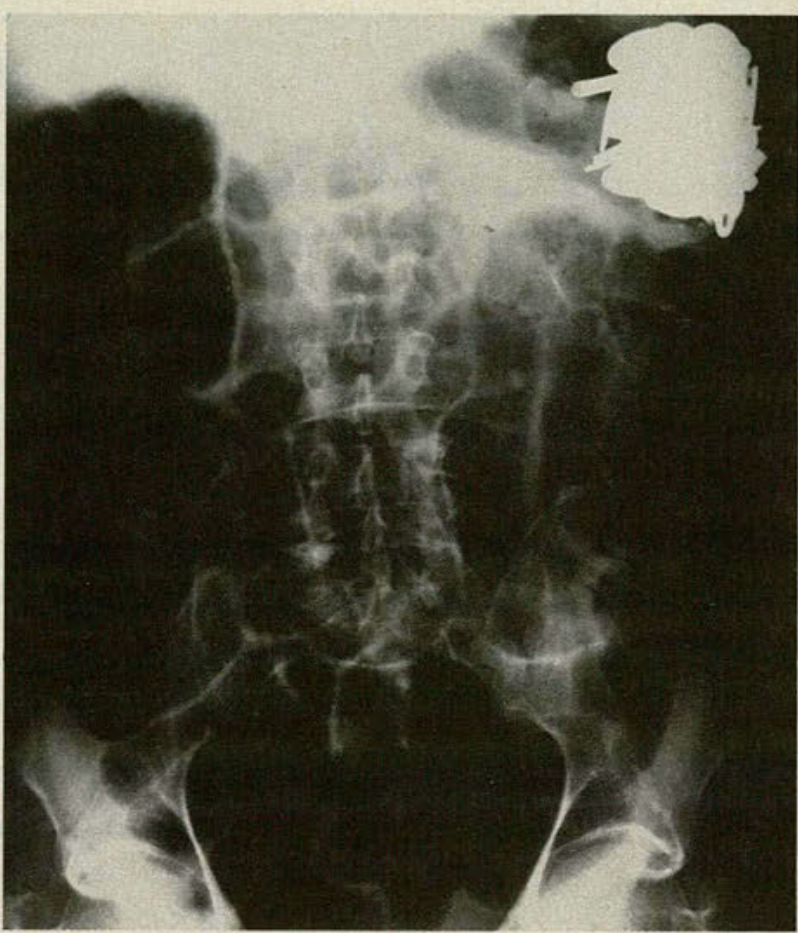

Figure 3. Plain abdominal $x$-ray showing gastric contents.

ety. He tolerated the endoscopic procedure well and was released.

\section{Discussion}

About 1500 people in the United States die of foreign body ingestion each year. ${ }^{3}$ Foreign body ingestion is common in psychiatric patients, elderly persons who wear dentures, children, suicidal patients, and malingering or manipulative patients.

The diagnosis of foreign body ingestion may be difficult to deduce from the patient's history, especially in children and psychotics, and when the ingestion may be remote. The diagnosis may be further complicated by the ingestion of radiolucent objects that cannot be seen on plain films without the use of a contrast medium.

Conservative treatment by watching and waiting should be the first approach to managing an ingested foreign body, because most such objects pass through the pylorus into the small bowel and are spontaneously excreted. 4 Foreign bodies that do not leave the stomach within 3 to 5 days should be removed endoscopically. Likewise, sharp objects such as needles and safety pins within the esophagus or 


\begin{tabular}{|rl|}
\hline \multicolumn{1}{|c|}{$\begin{array}{c}\text { Table } \\
\text { Gastric Contents }\end{array}$} \\
\hline 13 & Susan B. Anthony silver dollars \\
1 & John F. Kennedy half dollar \\
10 & quarters \\
9 & dimes \\
8 & nickels \\
14 & pennies \\
4 & metallic screws \\
2 & copper fittings \\
1 & saw fragment \\
4 & keys \\
1 & key chain \\
1 & charm bracelet with 2 charms \\
4 & metallic tags \\
2 & bolt fragments \\
4 & metallic nuts \\
1 & electrical connector \\
1 & large metallic staple \\
1 & drill bit \\
\hline
\end{tabular}

stomach that threaten perforation should probably be removed endoscopically. ${ }^{5}$

Surgery is indicated for large or long objects not clearing the stomach in 3 to 5 days, or when there is obstruction or perforation. ${ }^{6}$ Button batteries are usually not a problem; unless they are greater than $2 \mathrm{~cm}$ in diameter, they can be managed conservatively, with spontaneous passage likely. ${ }^{7}$ Cocaine trafficking has produced the so-called "body bagger" who will swallow packets of cocaine to prevent detection. If these packets rupture, the results can be fatal. Surgical intervention is required in this setting. ${ }^{8}$

In the past, heavy accumulations of foreign bodies were removed from the stomach surgically. Although larger collections of foreign objects have been reported, we believe that en-

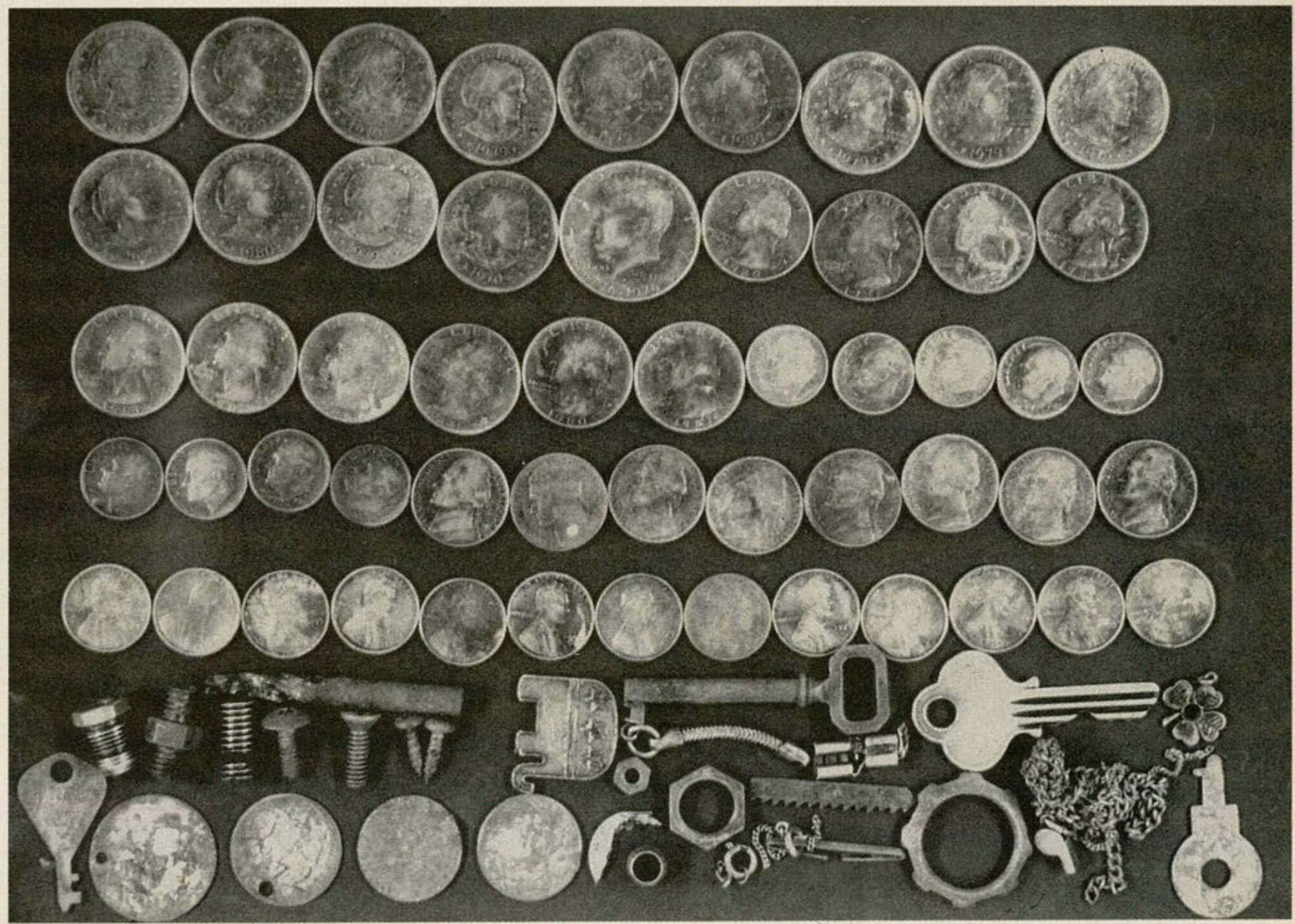

Figure 4. Removed gastric contents. Table provides inventory. 
doscopy, properly performed, is a safe and effective alternative to laparotomy. It should be now considered an alternative to surgery as a means of removing heavy accumulations of metallic foreign objects from the stomach when the objects are of appropriate size and can be removed safely.

\section{References}

1. DeBakey M, Ochsner A: Bezoars and concretions: Comprehensive review of literature with analysis of 303 collected cases and presentation of additional eight cases. Surgery 1938;4:934.
2. Chalk S, Foucal H: Foreign bodies in the stomach. Arch Surg 1928; $16: 494-500$

3. DeVanesan J, Pisani A, Pathanjali S, et al: Metallic foreign bodies in the stomach. Arch Surg 1977;112:664-665.

4. Nagendran T, Nagendran S: Foreign bodies of the gastrointestinal tract. J Med Assoc State Ala 1981;51:31-35.

5. Waye J: Removal of foreign bodies from the upper intestinal tract with fiberoptic instruments. Am $J$ Gastroenterol 1976;65:557-559.

6. Gracia C, Frey C, Balazs I: Diagnosis and management of ingested foreign bodies: A ten year experience. Ann Emer Med 1984;13:30-34.

7. Litovitz T: Button battery ingestions. JAMA 1983;249:24952500 .

8. Webb W: Techniques of foreign body removal: 'Tricks of the trade.' 1986 ASGE Postgraduate Course, San Francisco, Calif. 